\title{
COMMENTARY
}

\section{Hemofiltration or hemodialysis for acute kidney injury?}

\author{
Achim Jörres* \\ See related research by Friedrich et al., http://www.ccforum.com/content/16/4/R146
}

\begin{abstract}
The optimal choice of modality for acute renal replacement therapy is unclear at present. Diffusive therapy (hemodialysis) removes small solutes mainly, whereas convective therapies (hemofiltration and hemodiafiltration) may also eliminate larger molecules such as myoglobin or cytokines. Conversely, convective therapies might predispose patients to filter clotting and thus increased costs. A systematic review and meta-analysis of clinical trials could not find evidence for clinical benefits of either modality. Thus, the decision on renal replacement therapy modality still is based on the clinical status of the individual patient, the expertise of the medical and nursing staff, and local circumstances and availability.
\end{abstract}

The strategy for renal replacement therapy (RRT) in patients with acute kidney injury (AKI) remains controversial. To date, crucial questions such as the optimal timing, treatment intensity, and modality for acute RRT have not found definitive answers from adequately powered prospective randomized controlled trials (RCTs). An area of particular uncertainty is the choice between hemodialysis, hemofiltration, or hemodiafiltration (that is, between diffusive and convective treatment or a combination of the two).

Although all three modalities can easily be performed on a modern continuous RRT machine with a few simple modifications of the setup, some major technical differences do exist. With convection, the elimination of solutes across a membrane is carried by water flux, resulting in similar removal of small, middle-sized, and larger molecules for which the membrane is permeable. In contrast, diffusive clearance is highly efficient in

*Correspondence: achim.joerres@charite.de

Department of Nephrology and Medical Intensive Care, Charité University Hospital Campus Virchow-Klinikum, Augustenburger Platz 1, D-13353 Berlin, Germany removing small solutes but less so in eliminating larger compounds (such as uremic 'middle molecules', myoglobin, or cytokines). However, convection requires large rates of ultrafiltration in order to be effective. If the necessary replacement fluid is administered post-filter, hemoconcentration within the hemofilter will result and patients potentially will be predisposed to clotting. On the other hand, if replacement fluid is added pre-filter, the treatment efficacy is reduced since solutes are diluted before elimination.

Whether and how these fundamental differences translate into clinical outcomes are unclear at present. In this issue of Critical Care, Friedrich and colleagues [1] present a systematic review and meta-analysis of clinical trials that compare hemofiltration and hemodialysis for the treatment of AKI. The authors found no difference in mortality or other clinical outcomes such as RRT dependence in survivors, organ dysfunction, or vasopressor use. Not unexpectedly, the data suggested an increased clearance of larger molecules with hemofiltration but also a shorter filter life. The essential conclusions from the meta-analysis are that we do not have a sufficient database at present to recommend one procedure over the other, a fact reflected in the current AKI guidelines of the KDIGO (Kidney Disease Improving Global Outcomes) group [2] and the UK Renal Association [3], and that we will require larger clinical trials before definitive recommendations can be made.

However, the question is whether a 'definitive' prospective RCT in unselected populations with AKI will actually help to resolve this issue. As with the choice of intermittent versus continuous versus 'hybrid' RRT as the initial treatment for AKI, one size likely will not fit all. More likely, future studies will have to address the question of whether there are specific subgroups of patients who might benefit from convective therapies (for example, myoglobinuric or septic AKI patients in whom the enhanced removal of myoglobin or cytokines by hemofiltration might help to improve clinical course and renal recovery). Moreover, the question of RRT 'dose' is inextricably linked with the choice of modality. If replacement fluid is added pre-filter in order to limit 
hemoconcentration and clotting risk, total treatment volumes must be increased by $20 \%$ to $30 \%$ to achieve equivalent clearance of small solutes. This, together with a potentially reduced filter life, may lead to increased costs of convective therapies in comparison with continuous hemodialysis.

In clinical practice, the intensivist will have to balance the desired intensity of treatment, in particular with regard to the removal of larger molecules, against clotting risk, filter life, and costs. Inevitably, local experience and circumstances will also influence the choice of modality. As the current UK Renal Association guidelines [3] put it, 'choice of RRT modality should be guided by the individual patient's clinical status, medical and nursing expertise, and availability of modality'.

Abbreviations

$A K I$, acute kidney injury; $R C T$, randomized controlled trial; RRT, renal replacement therapy.

\section{Competing interests}

The author declares that he has no competing interests.

Published: 10 August 2012

\section{References}

1. Friedrich JO, Wald R, Bagshaw SM, Burns KE, Adhikari NJ: Hemofiltration compared to hemodialysis for acute kidney injury: systematic review and meta-analysis. Crit Care 2012, 16:R146.

2. Section 5: Dialysis interventions for treatment of AKI. In KDIGO Clinical Practice Guideline for Acute Kidney Injury. New York: Kidney Disease: Improving Global Outcomes (KDIGO). Kidney International Supplements 2012, 2:89-115.

3. Lewington A, Kanagasundaram S: UK Renal Association clinical practice guidelines acute kidney injury. Nephron Clin Pract 2011, 118 Suppl 1:c349-90.

doi:10.1186/cc11450

Cite this article as: Jörres A: Hemofiltration or hemodialysis for acute

kidney injury? Critical Care 2012, 16:147. 